\title{
Relationship Between Depression and Specific Health Indicators Among Hypertensive African American Parents and Grandparents
}

\author{
Jacquelyn Y. Taylor, PhD, APRN, BC, PNP, RN; ${ }^{1}$ Olivia G. M. Washington, PhD, APRN, BC, NP, LPC;2,3 \\ Nancy T. Artinian, PhD, RN, BC; ;,3 Peter Lichtenberg, PhD, ABPP 3
}

$\mathrm{N}$ egative lifestyle behaviors, including stress, obesity, lack of physical activity, sodium intake, and smoking, have been the focus of attention by government, news media, and the medical community. Negative lifestyle behaviors also can include the level of stress and support that may contribute to increased depressive symptoms among African American parents and grandparents. Research has shown that depression is directly influenced by stress, indicating that greater levels of stress can result in higher depression scores. ${ }^{1}$ Parental stress in particular has been noted to be significantly related to increases in diastolic blood pressure readings among African Americans. ${ }^{2}$ These stressors and lifestyle behaviors can be precursors to chronic diseases, including diabetes, hypertension, and cardiovascular disease.

Despite knowledge of complications of hypertension and well-recognized benefits of interventions that lower blood pressure, ${ }^{3,4}$ national surveys document suboptimal rates of hypertension awareness, treatment, and control particularly among African Americans. ${ }^{5-7}$ Improvements in the ability to recognize factors that may contribute to hypertension, such as depression, social support, and lifestyle, are essential to reducing the public health impact of hypertension and the disparities found among ethnic groups. Although an increasing number of studies have been published on psychosocial and lifestyle factors related to these health disparities, none have assessed the combined effects of personal characteristics, perceived levels

African Americans are at greater risk for hypertension than are other ethnic groups. This study examined relationships among hypertension, stress, and depression among 120 urban African American parents and grandparents. This study is a secondary analysis of a larger nurse-managed randomized clinical trial testing the effectiveness of a telemonitoring intervention. Baseline data used in analyses, with the exception of medication compliance, were collected at 3 month' follow-up. Health indicators, perceived stress, and social support were examined to determine their relationship with depressive symptoms. A total of $48 \%$ of the variance in depressive symptomology was explained by perceived stress and support. Health indicators including average systolic blood pressure explained 21\% of the variance in depressive symptomology. The regression analysis using average diastolic blood pressure explained 26\% of the variance in depressive symptomology. Based on study results, African Americans should be assessed for perceived stress and social support to alleviate depressive symptomology. Prog Cardiovasc Nurs. 2008;23:68-78. ${ }^{\circ} 2008$ Le Jacq

From the University of Michigan School of Nursing, Ann Arbor, MI; ${ }^{1}$ and College of Nursing ${ }^{2}$ and Institute of Gerontology, ${ }^{3}$ Wayne State University, Detroit, MI Address for correspondence: Jacquelyn Taylor, PhD, APRN, BC, PNP, RN, 400

North Ingalls, Room 3174, Ann Arbor, MI 48105

E-mail: jytaylor@umich.edu

Manuscript received November 13, 2007; revised January 28, 2008;

accepted March 5, 2008

of stress and support, and specific health indicators in understanding depression in hypertensive caregivers. The purpose of this study was to determine which personal characteristics, perceived levels of stress and support, and specific health indicators (ie, obesity, physical activity, sodium intake, medication compliance, and smoking) could be used to predict symptoms of depression among African American parents and grandparents with diagnosed hypertension.

\section{REVIEW OF LITERATURE}

\section{Stress and Depression}

Many studies have been conducted exploring the relationship between stress and depression. ${ }^{1,8-10}$ It has been shown that individuals who are unable to manage stress experience greater levels of depression. ${ }^{1}$ Stress appears to be a vital catalyst in the development of depression and the expression of other chronic diseases such as hypertension. ${ }^{9,10}$ Because stress can come from various sources and each individual's method of managing stress may differ, the order in which stress triggers depression and hypertension remains unclear.

Stress associated with caregiving may act as an additional burden to those already coping with the management of their own health issues, particularly depression or hypertension. Research has indicated that individuals lacking 
strong social support networks may experience caregiver stress that can be detrimental to their own psychological and physiologic health. ${ }^{10,11}$ This additional burden of caregiving may add to personal, social, and financial stress that could affect mood and blood pressure. ${ }^{2,12}$ Caregivers with low income may be faced with the choice of buying medication to care for themselves or providing necessities for their dependent child or grandchild. In these situations, parental stress and lack of adequate social support may contribute to symptoms of depression and alterations in blood pressure readings. $2,10,12,13$

\section{Hypertension and Depression Among African Americans}

African Americans have had a long tradition of providing kinship care. Culturally, caregiving within African American families is expected to be provided to children, elders, and even extended members of the family when needed. African American matriarchs sometimes become overextended in the multiple roles of wife, mother, grandmother, and caregiver. They often sacrifice their own self-care to meet the needs of others. This self-sacrifice among African American caregivers can result in negative physical and mental health outcomes. ${ }^{14}$ Researchers have found that African American caregivers who experience high levels of selfreported role strain and stress often experience poorer physical health and greater symptoms of depression. ${ }^{15}$ Although the prevalence of those with hypertension and depression is unclear, research has shown that African American adults typically have higher rates of hypertension (41.4\%) as compared with Caucasians (28.1\%), and African American women experience the highest rates of high blood pressure, at $44.0 \%$. $^{16}$

Investigators have found that symptoms of depression are linked to hypertension and the development of hypertension. ${ }^{17}$ This relationship has been established in younger ${ }^{18}$ and older adults. ${ }^{19}$ Jonas and Lando ${ }^{20}$ reported that Caucasian women and all men with increased negative affect had similarly elevated relative risks of hypertension in comparison with hypertensive African American women, who were at substantially higher relative risk for depressive symptomology. When Schnittker ${ }^{8}$ examined the link between high blood pressure and symptoms of depression in later life, participants who presented with chronic illnesses earlier in life were found to have more depressive symptoms in later life. These findings are important when considering risk for depression among hypertensive African American parents and grandparents.

\section{Depression and Parenting Roles}

Minkler and Fuller-Thomson ${ }^{21}$ reported that single African American grandmothers caring for grandchildren reported greater depressive symptoms than Caucasian grandmothers; however, no measurement of physical health status was given for grandmothers in the study. Musil and Ahmad ${ }^{22}$ reported that unemployed grandmothers caring for older grandchildren had more depressive symptoms than employed grandmothers caring for younger grandchildren. Their study revealed that high levels of parental stress were associated with greater depressive symptoms and lower levels of self-reported health status. However, another study failed to find a significant relationship between parental stress and physical health status of the caregiver but found that social factors (eg, lack of social activities, dysfunctional family relationships, and economic hardships) were significantly related to grandparents' health. ${ }^{11}$

Fuller-Thomson and Minkler ${ }^{23}$ conducted a national survey of African American grandparents raising grandchildren. Their findings revealed that caregivers who were more limited in activities of daily living had twice as many symptoms of depression as those whose activities of daily living were not limited. In contrast, findings from a study conducted by Whitley and colleagues $^{24}$ suggested that even when grandmothers caring for grandchildren have diminished physical health, their self-reported mental health status appeared to be equal to or greater than that of the general population.

\section{Specific Health Indicators and Depression}

Although researchers have examined depression, parental stress, and selfreported general health status among grandparent and parent caregivers, there is a paucity of research on the mechanisms most responsible for these conditions. In the current study, specific health indicators, such as (1) symptoms of depression; (2) obesity, body mass index (BMI), and physical activity; (3) dietary sodium, (4) cigarette smoking, (5) medication compliance, and (6) stress and social support, and their relationship to depression among hypertensive African American parents and grandparents were investigated.

Symptoms of Depression. Warren ${ }^{25}$ asserted that it is difficult to obtain information on the prevalence of depression in African American women because it may be misdiagnosed or they may withdraw from treatment when their ethnic and cultural needs are not met. African Americans generally do not report dysphoria, sleep changes, and thoughts of suicide. The most common symptom of depression in African American women is a change in appetite that results in weight gain. ${ }^{25}$ For the purpose of this study, depression is defined as the symptoms associated with depression, not a clinical diagnosis of depression.

Obesity, BMI, Physical Activity, and Depression. Several studies have established a positive relationship between obesity (BMI) and physical and mental health. ${ }^{26}$ Previous research indicated that obesity has been related to depression, with findings consistent when controlling for race. ${ }^{26,27}$ Obesity has been defined as BMI >30 mg/ $\mathrm{kg}^{2}$, with morbid obesity indicated by BMI $\geq 40 \mathrm{mg} / \mathrm{kg}^{2} \cdot{ }^{28,29}$ Elevation in body weight and BMI was linked with depression that could lead to body image disturbances. ${ }^{27} \mathrm{~A}$ significant positive relationship was found between obesity and depression, even 
after controlling for sex, race, chronic illnesses, family history of depression, and demographic risk factors. ${ }^{30}$

The exact physiologic mechanism linking physical activity and mood alteration is still unknown, but speculation has been raised that exercise can increase secretion of catecholamine that can elevate mood. ${ }^{31}$ Research has shown that aerobic and anaerobic physical activity in intervals of 10 to 15 minutes contributes to substantial positive mood changes. ${ }^{32}$ Research has also indicated that physical activity resulting in small declines in weight or body fat can elevate mood and overall quality of life among patients diagnosed with hypertension. ${ }^{33}$

Dietary Sodium and Depression. A consistent diet of food high in sodium has been shown to be detrimental to physical and mental health.9,34 Excessive sodium intake can lead to water retention that can elevate both body weight and blood pressure levels. In many cases, African Americans consume high-sodium, high-cholesterol foods as a basis in their daily diets, which can lead to obesity, high blood pressure, and depression. ${ }^{30}$ Culturally, African Americans, when depressed, may overeat and use food as a means of comfort. ${ }^{35}$ However, a link has not been established between sodium intake and depression in African Americans.

Celebration, comfort, and family gatherings through cooking and eating soul foods (eg, fried chicken, fried pork chops, chitterlings, collard greens, candied yams, macaroni and cheese) rich in salt and cholesterol have been a cultural norm for some African Americans. Many African American women pride themselves on being able to prepare rich soul food dishes from recipes handed down by the women in their families. These foods are prepared to elevate moods and provide a sense of pride in having the capacity to feed and provide for the entire household, ${ }^{12,21}$ thus perpetuating high sodium intake and the increased probability of hypertension.
Cigarette Smoking and Depression. Results of the examination of cigarette smoking and depression in persons with diagnosed hypertension as reported in the Coronary Artery Risk Development in Young Adults (CARDIA) study were mixed. ${ }^{36}$ When smokers were compared with nonsmokers, smokers with a dependence on nicotine had significantly higher occurrences of both depression and hypertension. The CARDIA study also found that African American women smokers with low educational levels were more likely to be depressed. Repetto and associates ${ }^{37}$ indicated that depression earlier in life among African Americans predicted cigarette use as a means to stabilize mood. In contrast, a study examining depression and cigarette smoking among low-income African Americans residing in a public housing community concluded that cigarette smoking was unrelated to symptoms of depression. ${ }^{38}$

Hypertensive Medication and Depression. A myriad of medications are available for hypertension management, including angiotensinconverting enzyme inhibitors, angiotensin II receptor blockers, $\beta$-blockers, calcium channel blockers, and thiazide diuretics. However, other studies have suggested that combination pharmacologic therapy (prescribing $>1$ hypertension drug) can have greater effectiveness than monotherapy in African Americans. ${ }^{34}$ Hypertension medications, particularly angiotensinconverting enzyme inhibitors, have been shown to elevate mood and decrease symptoms of depression. ${ }^{39}$ The researchers found that participants who received captopril and/or enalapril had fewer occurrences of depression than participants who received other hypertensive medications. In addition, hypertensive patients who did not receive these medications had higher arterial blood pressure and significantly higher occurrences of depression.

Stress and Social Support. Lack of a social support network may intensify some stress that individuals internalize, leading to declines in physical and mental health. The majority of African American women are the head of families and often lack support systems that can help reduce stress. ${ }^{2}$ These women must contend with living in a male-dominated society that often devalues their African American ethnicity, culture, and sex. While many are living at lower socioeconomic levels, some are becoming stressed as they try to improve their family's standing in mainstream society. ${ }^{40}$ These women assume multiple caregiving roles as mother, daughter, and significant other. Caregiving burdens may place additional stress on persons with chronic health conditions, especially among those who lack support systems. According to Warren, ${ }^{25}$ these factors can intensify stress that erodes self-esteem, social support systems, and caregiver health.

Role of Religion and Stress. A study of a nationally representative sample of African Americans found that they reported higher levels of religious participation than non-Hispanic whites as a method of coping with stress and depression. ${ }^{41}$ The use of religious beliefs as a coping mechanism against stress was linked with desired outcomes in stroke patients and appeared to be a factor in improved self-confidence and positive outlook. ${ }^{42}$ Other research has shown that participation in religious activities can be beneficial in improving a patient's ability to cope with stress. The use of religion as a coping strategy has been linked with positive health outcomes, such as lowered blood pressure. ${ }^{43}$ Because participation in religious activity is a prominent part of African American culture, ${ }^{41}$ assessing the use of religion as a coping mechanism in dealing with stress, depression, and high blood pressure is important.

\section{PURPOSE OF THE STUDY}

The purpose of this study was to determine which personal characteristics, perceived levels of stress and support, and specific health indicators (obesity, 
physical activity, sodium intake, medication, and smoking) could be used to predict symptoms of depression among African American parents and grandparents with diagnosed hypertension. The following research questions were addressed:

1. Can symptoms of depression be predicted from personal characteristics and perceived levels of stress and social support?

2. Can symptoms of depression be predicted from specific health indicators, including systolic and diastolic blood pressure, obesity represented by BMI, physical inactivity, dietary sodium intake, taking prescribed antihypertensive medication, cigarette smoking, and selfreported opinion of health?

\section{METHODS}

The present study used a correlational research design. The study was nested within a longitudinal randomized clinical trial designed to test effects of a telemonitoring intervention of African Americans with hypertension over a 1 -year period. Results of the telemonitoring intervention can be found elsewhere. ${ }^{44}$ Participants in the present study were a subgroup of hypertensive African Americans from the parent study who had caregiving responsibility for children and/or grandchildren. Data associated with all study variables were collected at baseline, with the exception of compliance to hypertensive medication regimen. The Wayne State University Human Investigation Committee approved the study protocol for both the parent study and the present study. Prior written informed consent was obtained before participation in the parent study.

\section{Sampling Procedures}

The inclusion criteria for participants in the parent study were diagnosis of at least stage I hypertension (systolic blood pressure $\geq 140 \mathrm{~mm} \mathrm{Hg}$ or diastolic blood pressure $\geq 90 \mathrm{~mm} \mathrm{Hg}$, or if diabetic, systolic blood pressure $>130 \mathrm{~mm} \mathrm{Hg}$ or diastolic blood pressure $>80 \mathrm{~mm} \mathrm{Hg}$ ), African American ethnicity, and ability to read and understand English. Exclusion criteria were active substance abuse, history of mental illness other than depression, end-stage cancer, end-stage renal disease, and other terminal illnesses.

The sample included 120 African American participants from the parent study. Findings of a power analysis indicated that a multiple regression with 7 variables requires 97 to 102 participants to generate a power of 0.80 at an $\alpha$ level of .05 with a moderate effect size. ${ }^{45}$ Participants $(\mathrm{N}=120)$ who met the inclusion criteria of the parent study and reported having parenting/grandparenting responsibility were included in the present study. Study participants were compensated $\$ 25$ for 2 hours of their time spent in data collection.

\section{Measurements}

Demographic Survey. Demographic questions included caregiving, caregiving relationship, level of education, age, sex, marital status, and total household income. The demographic survey was designed to collect information from participants regarding their caregiving relationships with their children, adult children with disabilities, or grandchildren. ${ }^{13}$ Other information collected included number of children for whom participants were currently caring, number of hours per week spent caregiving, legal relationship with the child receiving care, and existence of cognitive or physical disabilities. ${ }^{13}$

Participants were asked to selfreport their health using a 5-point scale ranging from excellent to poor. In addition, they were asked whether they smoked cigarettes. Their response to this question was either yes or no.

Stress and Social Support Section. Stress and social support were measured using 7 questions from the protocol used in the parent study. Four items measured stress and the remaining 3 items obtained data on social support.

A 4-item scale drawn from the Perceived Stress Scale was used to measure stress. ${ }^{46}$ Two items focused on the extent to which stressful situations were occurring in one's life, and frequency was scored on a scale from 1 (never) to 5 (very often). The third item examined perceived control over stressful situations and was scored on a scale of 0 (complete control) to 6 (no control). A fourth item examined the respondents' perception of their ability to decrease their stress and was rated using a scale of 0 (can decrease completely) to 6 (cannot decrease). Each item was considered a separate variable in this study. The internal consistency of the scale as a measure of reliability was 0.78 and had good construct validity. ${ }^{46}$ Cronbach's $\alpha$ reliability for the scale was .85 in the parent study. ${ }^{9}$

Three items from the ENRICHD Social Support Instrument (ESSI) were used to measure available emotional support that parents and grandparents perceived they received. ${ }^{47}$ The items were rated using a 5-point Likerttype scale ranging from 1 (none of the time) to 5 (all of the time). The items measuring social support were used as separate independent variables in the present study. The ESSI has been found to have acceptable internal consistency and a reliability of $0.86 .{ }^{48}$ Cronbach's $\alpha$ reliability of the ESSI was .86 in the parent study. ${ }^{?}$

\section{Center for Epidemiologic Studies-} Depression Scale. The Center for Epidemiologic Studies-Depression Scale (CES-D) was developed to measure the frequency with which individuals experienced depressive symptomology. The CES-D is not intended to be used as a diagnostic tool or to evaluate the severity of depression over the course of treatment. ${ }^{49}$ The instrument comprises 20 items that measure major depressive symptoms that have been identified in clinical literature. The items on the CES-D originally were rated using a 4-point scale. ${ }^{9}$ In the parent study, the items were measured using a 5-point Likerttype scale ranging from 1 for none of the time to 5 for all of the time to measure the frequency with which the symptoms occurred over the past 
7 days. Individual scores were summed to produce a possible total score ranging from 20 to 100 . A score of 42 was determined to be mathematically equivalent to the cutoff score of 16 on the original instrument, which is suggestive of clinical depression. The CES-D has been used extensively in research and has excellent reliability and validity. ${ }^{49}$ Reliability of the CES-D in the parent study was .93. ${ }^{9}$

Blood Pressure. Blood pressure readings were taken according to Joint National Committee guidelines using an electronic blood pressure monitor (Omron HEM-737 Intellisense, Omron Healthcare, Inc, Vernon Hills, IL) that has been validated by the British Hypertension Society and the Association for the Advancement of Medical Instrumentation. ${ }^{50,51}$ Blood pressure levels were measured at least twice, and separate averages for diastolic and systolic blood pressure readings were used for analyses.?

Obesity. BMI is the relationship between weight and height that is associated with body fat and health risk. The formula for calculating BMI is weight in pounds divided by the height in inches squared. ${ }^{28}$ The result is then multiplied by 703 to obtain the BMI. BMI $>25 \mathrm{mg} / \mathrm{kg}^{2}$ is considered overweight, and BMI $\geq 30 \mathrm{mg} / \mathrm{kg}^{2}$ is indicative of obesity. An electronic scale (BWB/807 Tanita, Tokyo, Japan) was used to measure weight, and height was measured using a portable stadiometer (Model 214 Road Rod, Seca Corporation, Hanover, MD).

Physical Activity. The recommendation for physical activity is 30 minutes each day for patients with diagnosed hypertension. ${ }^{50}$ To measure physical activity, participants were asked 2 questions. Participants were asked whether they took part in a minimum of 30 minutes of moderate or greater physical activity on any day in the past 7 days. They were then asked to indicate the number of minutes of participation in moderate or greater physical activity over the past 7 days. The minutes of moderate or greater physical activity were used for analyses in this study.

Sodium Intake. Sodium intake (ie, mg of sodium per day) was determined using self-reported data from 24-hour food recalls. Data were translated into nutrient intakes using computer software entitled the Food Processor (ESHA Research, Salem, OR). Multiple-day food recall is considered valid because it represents a person's usual or habitual intake accurately. ${ }^{52}$ Participants were contacted on 2 randomly selected weekdays and 1 weekend day to ask them to recall what they had eaten within the last 24 hours. Each recall took approximately 10 minutes to complete. Food models and measuring cups and spoons were used as memory aids to assist participants in reporting accurate serving sizes. Information on sodium intake that was collected at the baseline interview was obtained from the parent study.

Percentage of Antibypertensive Medication Doses Taken as Prescribed. Medication compliance was measured using an electronic drug exposure monitoring device (MEMS caps versions 5 and 6). ${ }^{44}$ The lid of this bottle contained a microchip that collected information regarding dates and times that the bottle had been opened and closed. Participants were asked to open the bottle only when taking their antihypertensive medication and to refill the bottle only when taking their regularly scheduled doses of medication. Other medications were not measured. The parent study was designed for tracking one medication using the MEMS caps device. Although it is not unusual for multiple medications to be taken for hypertension control, only one medication was monitored by the MEMS caps. One MEMS caps device was provided for each participant. If the participant was taking other medication, the name and dosage of other medications were recorded during the structured interviews. The MEMS caps device recorded daily compliance with use of one antihypertensive medication only. Information on medication compliance was obtained from the parent study. This information was collected at the 3-month follow-up. ${ }^{44}$ In the current study, participants' general medication compliance was evaluated by determining whether they took their medication as prescribed (eg, 1, 2, or 3 times a day), resulting in a percentage of medication doses taken daily.

\section{Statistical Analysis}

Descriptive statistics were used to provide a personal profile of sample participants. Stepwise multiple linear regression analyses were used to test each of the hypotheses, with all decisions on the statistical significance of the outcomes made using a criterion $\alpha$ level of .05.

\section{RESULTS}

\section{Description of Sample}

Personal Characteristics. Participants' ages in the sample ranged from 22 to 82 years, with a mean of $54.03 \pm 13.15$ years. Most participants identified their sex as female ( $n=83 ; 69.2 \%)$. The largest group of participants $(\mathrm{n}=41 ; 34.5 \%)$ had completed high school, with $37(31.1 \%)$ indicating that they had some college experience. Thirty-nine $(32.5 \%)$ participants were single/never married, with 31 (25.8\%) reporting that they were married. The majority of participants indicated they were not working $(n=72 ; 60.0 \%)$ and reported annual incomes $<\$ 20,000$ $(\mathrm{n}=74 ; 62.7 \%)$ (Table I).

Family Characteristics. The largest group of parents/grandparents had 1 child ( $\mathrm{n}=67 ; 55.8 \%)$, with 44 (36.7\%) reporting 2 children living in their homes. Fifty-nine (49.2\%) of the participants were caring for their grandchildren, and 61 (50.8\%) were responsible for their own children. Most participants ( $\mathrm{n}=71 ; 59.2 \%)$ were providing child care 24 hours a day, 7 days a week. Nine $(7.5 \%)$ parents/grandparents had children who were physically/ mentally disabled, and 51 (42.5\%) had legal custody of their children/ 
grandchildren. Sixty-one (50.8\%) parents/grandparents reported that they practiced religious activities to relieve stress. Participants who engaged in religious activities spent from 1 to 5 hours a week participating in these activities $(\mathrm{n}=40 ; 65.6 \%)$ (Table II).

\section{Descriptive Health Indicator}

Statistics. Descriptive statistics were used to summarize participants' health indicators. The average systolic blood pressure level was $115.47 \pm 20.40 \mathrm{~mm}$ $\mathrm{Hg}$, with a range from 113 to 209 $\mathrm{mm} \mathrm{Hg}$. The mean diastolic blood pressure level was $91.57 \pm 14.00 \mathrm{~mm}$ $\mathrm{Hg}$ and ranged from 61 to 128 $\mathrm{mm} \mathrm{Hg}$. Participants had a mean BMI of $32.63 \pm 7.09 \mathrm{mg} / \mathrm{kg}^{2}$, with a range from 18.27 to $64.22 \mathrm{mg} / \mathrm{kg}^{2}$. Average sodium intake at baseline was $2456.23 \pm 1166.88 \mathrm{mg}$ and ranged from 189.33 to $6130.00 \mathrm{mg}$. The patients' medication regimens were measured by the percentage of prescribed medication doses taken. The mean percentage was $28.8 \% \pm 38.9 \%$. The range of medication doses taken was from $0 \%$ to $107.8 \%$ (percentages $>100 \%$ indicated that participants were taking more doses than prescribed). The minutes per week of physical activity in which the parents/ grandparents participated ranged from 98.75 to 211 minutes, with a mean of $156.81 \pm 23.36 \mathrm{~min}$ (Table III).

\section{Personal Characteristics and Perceived Stress and Social Support Analysis}

Four predictor variables (how often have you felt overwhelmed by problems?, how often do you feel stressed?, can you decrease your stress?, and level of education) entered the stepwise multiple linear regression equation, explaining a statistically significant amount of variance in depression symptomology, $r^{2}=0.48 ; P<.001$. The negative relationships indicated that higher scores on depression symptomology were related to greater stress. Parents/grandparents with higher levels of education were less likely to be
Table I. Personal Characteristics of Participants

\begin{tabular}{|c|c|c|}
\hline Personal Characteristics $(\mathrm{N}=120)$ & No. & $\%$ \\
\hline \multicolumn{3}{|l|}{ Age, $y^{\mathrm{a}}$} \\
\hline$<30$ & 3 & 2.5 \\
\hline $31-40$ & 15 & 12.5 \\
\hline $41-50$ & 31 & 25.9 \\
\hline $51-60$ & 37 & 30.8 \\
\hline $61-70$ & 18 & 15.0 \\
\hline$>70$ & 16 & 13.3 \\
\hline \multicolumn{3}{|l|}{ Sex } \\
\hline Male & 37 & 30.8 \\
\hline Female & 83 & 69.2 \\
\hline \multicolumn{3}{|l|}{ Education level ${ }^{\mathrm{b}}$} \\
\hline Less than high school & 6 & 5.0 \\
\hline Some high school & 23 & 19.3 \\
\hline High school diploma/GED & 41 & 34.5 \\
\hline Some college & 37 & 31.1 \\
\hline Bachelor degree & 7 & 5.9 \\
\hline Graduate school & 5 & 4.2 \\
\hline \multicolumn{3}{|l|}{ Marital status } \\
\hline Single/never married & 39 & 32.5 \\
\hline Married & 31 & 25.8 \\
\hline Divorced & 23 & 19.2 \\
\hline Widowed & 16 & 13.3 \\
\hline Separated & 11 & 9.2 \\
\hline \multicolumn{3}{|l|}{ Employment status } \\
\hline Working & 48 & 40.0 \\
\hline Not working & 72 & 60.0 \\
\hline \multicolumn{3}{|l|}{ Total household income } \\
\hline$<\$ 5,000$ & 17 & 14.4 \\
\hline$\$ 5,000-\$ 9,999$ & 25 & 21.2 \\
\hline$\$ 10,000-\$ 19,999$ & 32 & 27.1 \\
\hline$\$ 20,000-\$ 29,999$ & 11 & 9.3 \\
\hline$\$ 30,000-\$ 49,999$ & 16 & 13.6 \\
\hline$\geq \$ 50,000$ & 8 & 6.8 \\
\hline Refused & 6 & 5.1 \\
\hline Did not know & 3 & 2.5 \\
\hline
\end{tabular}

depressed than those with lower levels of education (Table IV).

\section{Depression and Health Indicators} Analysis: Systolic Blood Pressure With depression as the dependent variable, systolic and diastolic blood pressure values were analyzed as independent variables in separate stepwise linear regressions. Systolic blood pressure was included in the first analysis and diastolic blood pressure in the second analysis to reduce the effects of multicollinearity. In the systolic blood pressure analysis, 4 variables (physical activity, self-reported opinion of health, average systolic blood pressure, smoking at time of study) explained $21 \%$ of the variance. With the exception of smoking at the time of the study, the relationships were in a positive direction, indicating that higher 


\begin{tabular}{|c|c|c|}
\hline FAMILY CHARACTERISTICS $(\mathrm{N}=120)$ & No. & $\%$ \\
\hline \multicolumn{3}{|l|}{ Relationship to child } \\
\hline Grandparent & 59 & 49.2 \\
\hline Parent & 61 & 50.8 \\
\hline \multicolumn{3}{|l|}{ No. of children in home } \\
\hline 1 & 67 & 55.8 \\
\hline 2 or 3 & 44 & 36.7 \\
\hline 4 or 5 & 4 & 3.3 \\
\hline$\geq 6$ & 5 & 4.2 \\
\hline \multicolumn{3}{|c|}{ No. of hours caring for children per week } \\
\hline$<30$ & 28 & 23.3 \\
\hline $30-49$ & 16 & 13.3 \\
\hline $50-69$ & 5 & 4.2 \\
\hline$\geq 70$ & 71 & 59.2 \\
\hline \multicolumn{3}{|c|}{ Child is physically/mentally disabled } \\
\hline Yes & 9 & 7.5 \\
\hline No & 111 & 92.5 \\
\hline \multicolumn{3}{|l|}{ Legal custody of child } \\
\hline Yes & 51 & 42.5 \\
\hline No & 69 & 57.5 \\
\hline \multicolumn{3}{|c|}{ Practice religious activities to relieve stress } \\
\hline Yes & 61 & 50.8 \\
\hline No & 59 & 49.2 \\
\hline \multicolumn{3}{|c|}{$\begin{array}{l}\text { No. of hours spent in religious activities per } \\
\text { week }(n=61)\end{array}$} \\
\hline $1-5$ & 40 & 65.6 \\
\hline $6-10$ & 13 & 21.3 \\
\hline $11-15$ & 2 & 3.3 \\
\hline$>15$ & 6 & 9.8 \\
\hline
\end{tabular}

scores on depression were associated with increased physical activity and average systolic blood pressure and lower self-reported opinions of health. Participants who were not smoking at the time of the study had lower levels of depression (Table V).

\section{Depression and Health Indicators Analysis: Diastolic Blood Pressure} In the analysis using diastolic blood pressure, 4 variables (average diastolic blood pressure, physical activity, selfreported opinion of health, smoking at time of the study) explained $26 \%$ of the variance. The positive relationship between depression and the independent variables (except for smoking at the time of the study) indicated that participants with higher depression scores were likely to have higher diastolic blood pressure, increased physical activity, and lower self-reported opinion of health. The negative relationship between depression and smoking at the time of study indicated that participants who were not smoking at the time of the study were likely to have lower levels of depression (Table VI).

\section{DISCUSSION}

Living with chronic hypertension can be stressful for those with the diagnosis and their families. Making lifestyle changes with limited financial and sociocontextual resources can be difficult. African Americans who are responsible for caring for children and/or grandchildren may experience parental stressors that could impact their blood pressure and mood. These factors could negatively influence expectations of quality of life for parents and grandparents.

This study supports claims that among parenting families, personal and social variables, such as feelings of stress and lower levels of education, could predict higher symptoms of depression. Health indicators (eg, high systolic and diastolic blood pressure and low self-reported opinion of health rating) also appear to be predictors of greater symptoms of depression among hypertensive African American parents and grandparents. These findings support previous research on the statistically significant relationship between hypertension and depression in African Americans. ${ }^{9,10}$

Findings of this study revealed that participation in physical activity was negatively related to depression. These findings conflict with previous research on physical activity and depression. ${ }^{30-32}$ One explanation for this inconsistency is that many depression studies have focused on mental health problems that could precede or predict physiologic health issues, such as complications with hypertension. This study, however, paid special attention to alternative social and health factors that could influence symptoms of depression. Another possible explanation is that patients may have tended to overreport their involvement with physical activity either because they misunderstood the question when asked or because they did not want to appear to be noncompliant with recommendations for physical activity.

The sodium intake measure was obtained from self-report of food intake. The amount of sodium included in the reported foods was estimated using a computer program. Some of the participants indicated that they ate soul foods that typically are high in sodium content. Most soul food is prepared at home from recipes passed down in families. As a result, the sodium content may not be readily calculated or compared using a computer program that attempts to standardize the amount of sodium in a specific food. 


\begin{tabular}{|lrrrrrr|}
\hline Table III. Health Indicators \\
\hline & \multicolumn{1}{l}{ No. } & \multicolumn{1}{c|}{ MEAN } & \multicolumn{1}{c|}{ SD } & MEDIAN & MINIMUM & MAXIMUM \\
HEALTH INDICATOR & 105 & 115.47 & 20.40 & 153.00 & 113.00 & 209.00 \\
\hline Average systolic blood pressure, $\mathrm{mm} \mathrm{Hg}$ & 105 & 91.57 & 14.00 & 92.00 & 61.00 & 128.00 \\
Average diastolic blood pressure, $\mathrm{mm} \mathrm{Hg}$ & 118 & 32.63 & 7.09 & 32.02 & 18.27 & 64.22 \\
Body mass index, $\mathrm{mg} / \mathrm{kg}^{2}$ & 119 & 156.81 & 23.36 & 160.13 & 98.75 & 211.00 \\
Weekly physical activity, min & 95 & 2456.23 & 1166.88 & 2228.00 & 189.33 & 6130.00 \\
Sodium intake at baseline, mg & 71 & 28.80 & 38.91 & 2.20 & 0.00 & 107.80 \\
Percentage of prescribed medication & & & & & & \\
$\quad$ doses taken & 120 & 1.72 & .45 & 2.00 & 1.00 & 2.00 \\
Cigarette smoking & 120 & 3.27 & .83 & 3.00 & 1.00 & 5.00 \\
Self-reported opinion of health & & & & & & \\
\hline
\end{tabular}

Table IV. Stepwise Multiple Linear Regression Analysis: Depression With Personal Characteristics, Perceived Stress, and Support

\begin{tabular}{|c|c|c|c|c|c|c|c|}
\hline \multicolumn{2}{|c|}{ PREDICTOR VARIABLES } & CONSTANT & B-WEIGHT & $\beta$ & $\delta R^{2}$ & T-VALUE & SIG OF T \\
\hline \multicolumn{8}{|c|}{ Included Variables } \\
\hline \multicolumn{2}{|c|}{$\begin{array}{l}\text { How often have you felt overwhelmed by } \\
\text { problems? }\end{array}$} & 4.01 & -.16 & -.30 & .36 & -3.10 & .002 \\
\hline \multicolumn{2}{|c|}{ How often do you feel stressed? } & & -.18 & -.27 & .06 & -3.00 & .003 \\
\hline \multicolumn{2}{|c|}{ Can you decrease your stress? } & & -.13 & -.22 & .04 & -2.58 & .011 \\
\hline \multicolumn{2}{|c|}{ Level of education } & & -.04 & -.15 & .02 & -2.10 & .038 \\
\hline \multicolumn{8}{|c|}{ Excluded variables } \\
\hline \multicolumn{2}{|l|}{ Age } & & & -.01 & & -.17 & .867 \\
\hline \multicolumn{2}{|l|}{ Sex } & & & -.11 & & -1.48 & .141 \\
\hline \multicolumn{2}{|c|}{ Total household income } & & & .08 & & 1.06 & .291 \\
\hline \multicolumn{2}{|c|}{ Present marital status } & & & $<.01$ & & -.01 & .996 \\
\hline \multicolumn{2}{|c|}{ Are you working now? } & & & -.04 & & -.48 & .631 \\
\hline \multicolumn{2}{|c|}{ How much stress control do you have? } & & & .02 & & .22 & .825 \\
\hline \multicolumn{2}{|c|}{ Can you count of someone to listen? } & & & -.05 & & -.62 & .539 \\
\hline \multicolumn{2}{|c|}{ Someone available to give good advice } & & & -.04 & & -.47 & .643 \\
\hline \multicolumn{2}{|c|}{$\begin{array}{l}\text { Someone available to help with daily } \\
\text { chores }\end{array}$} & & & -.09 & & -1.19 & .236 \\
\hline \multicolumn{2}{|c|}{ No. of children/grandchildren in home } & & & -.02 & & -.25 & .806 \\
\hline \multicolumn{2}{|c|}{ Revised relationship to child } & & & -.13 & & -1.83 & .069 \\
\hline \multicolumn{2}{|c|}{ Child is physically/mentally disabled } & & & .03 & & .41 & .680 \\
\hline \multicolumn{2}{|c|}{ Legal custody of children/grandchildren } & & & .13 & & 1.78 & .077 \\
\hline \multicolumn{2}{|c|}{ Practice religious activities to relieve stress } & & & .05 & & .76 & .451 \\
\hline Multiple $r$ & 0.69 & & & & & & \\
\hline Multiple $r^{2}$ & 0.48 & & & & & & \\
\hline$F$ ratio & 24.62 & & & & & & \\
\hline & 4,109 & & & & & & \\
\hline Sig of $F$ & $<.001$ & & & & & & \\
\hline
\end{tabular}

Another consideration is that the timing of the food recalls reflect what the person ate in the last 24 hours. Some of these persons may have been aware that they would be asked about their dietary intake and adjusted their consumption (or their reports) to reflect a socially desirable response.
Smoking has been linked to hypertension and depression in previous research that has found that individuals with depression use tobacco as a mood stabilizer. ${ }^{36}$ Other studies have noted that socioeconomic status may also result in persons using tobacco to self-medicate the effects of depressive symptoms. ${ }^{37,38}$ In the present study, smoking was a statistically significant predictor of depressive symptoms among hypertensive parents and grandparents. While the use of cigarettes and other tobacco products has been related to unhealthy lifestyle choices in both research and 


\begin{tabular}{|c|c|c|c|c|c|c|c|}
\hline \multicolumn{2}{|c|}{ PREDICTOR VARIABLES } & CONSTANT & B-WEIGHT & $\beta$ & $\delta R^{2}$ & T-VALUE & SIG OF T \\
\hline \multicolumn{8}{|c|}{ Included Variables } \\
\hline \multicolumn{2}{|c|}{ Physical activity } & -.75 & .01 & .28 & .08 & 3.30 & .001 \\
\hline \multicolumn{2}{|c|}{ Self-reported opinion of health } & & .17 & .20 & .06 & 2.34 & .021 \\
\hline \multicolumn{2}{|c|}{ Average systolic blood pressure } & & .01 & .21 & .03 & 2.49 & .014 \\
\hline \multicolumn{2}{|c|}{ Smoking at time of study } & & -.32 & -.20 & .04 & -2.36 & .020 \\
\hline \multicolumn{8}{|c|}{ Excluded variables } \\
\hline \multicolumn{2}{|c|}{ Body mass index } & & & .02 & & .17 & .866 \\
\hline \multicolumn{2}{|c|}{ Sodium intake, $\mathrm{mg}$} & & & .01 & & 15 & .880 \\
\hline \multicolumn{2}{|c|}{ Percentage of prescribed mediation does taken } & & & .03 & & .30 & .761 \\
\hline Multiple $r$ & 0.46 & & & & & & \\
\hline Multiple $r^{2}$ & 0.21 & & & & & & \\
\hline$F$ ratio & 7.68 & & & & & & \\
\hline$D F$ & 4,115 & & & & & & \\
\hline Sig of $F$ & $<.001$ & & & & & & \\
\hline
\end{tabular}

\begin{tabular}{|c|c|c|c|c|c|c|c|}
\hline Predictor & BLES & CONSTANT & B-WEIGHT & $\beta$ & $\delta R^{2}$ & T-VALUE & SIG OF T \\
\hline \multicolumn{8}{|c|}{ Included variables } \\
\hline Average & lic blood pressure & -1.23 & .02 & .30 & .08 & 3.66 & $<.001$ \\
\hline Physical & & & .01 & .30 & .10 & 3.72 & $<.001$ \\
\hline Self-repo & spinion of health & & .17 & .19 & .05 & 2.33 & .022 \\
\hline Smoking & ne of study & & -.27 & -.17 & .03 & -2.08 & .040 \\
\hline \multicolumn{8}{|c|}{ Excluded variables } \\
\hline Body ma & & & & -.01 & & -.11 & .912 \\
\hline Sodium i & mg & & & -.03 & & -.32 & .747 \\
\hline Percentas & nedication doses taken as prescribed & & & .07 & & .78 & .435 \\
\hline \multicolumn{8}{|c|}{ Multiple $r \quad 0.51$} \\
\hline Multiple $r^{2}$ & 0.26 & & & & & & \\
\hline$F$ ratio & 9.85 & & & & & & \\
\hline$D F$ & 4,115 & & & & & & \\
\hline Sig of $F$ & $<.001$ & & & & & & \\
\hline
\end{tabular}

popular literature, the links among cigarette smoking, hypertension, and depression are not clear and should be the focus of additional research.

African Americans are at higher risk for hypertension, and generally they receive less medical treatment than persons of other races. ${ }^{16}$ Because hypertension is a silent disease, many of these individuals may not be aware of their conditions and fail to seek medical treatment. Once a person has signs and/or symptoms of hypertension, through either a heart attack or stroke, organ damage may have already occurred. After a serious cardiovascular event, depression may become a secondary condition that can seriously affect a person's life. Individuals who experience depression may have greater difficulty adhering to their hypertension regimen that includes medication, physical exercise, reduced sodium intake, smoking cessation, and maintenance of a social support system.

\section{Strengths and Limitations}

The strength of this study is the addition to the knowledge base about the relationships among hypertension and depression and physical activity. The relationships among depressive symptomology, perceived stress, social support, education, and specific health indicators are important findings that can provide researchers and clinicians with knowledge on how to help patients become more proactive in their treatment of hypertension and depression.

Data used for this study had been collected as part of a larger parent study. The researcher lacked control over the data collection process. The study was limited to 120 parent study participants who had parenting responsibilities. Results cannot be generalized to other parent study participants who did not have similar responsibilities. The parent study was limited to African Americans who 
were living in a large urban area in the Midwest. African American parents and grandparents who live in suburban or rural areas may have different outcomes and experiences than those who live in urban areas.

The use of subjective measures (eg, physical activity, sodium intake, past and present smoking) may have been areas of bias that could affect the study outcomes. Participants in the study may have given responses that were socially acceptable, instead of providing accurate accounts of these variables. In addition, pack/year history of smoking and history of quitting smoking were not examined, and these questions may provide insight into residual effects of smoking. Researchers for the parent study altered the rating scale for CES-D. 'This change may have limited comparison of the results, as the summated scores in the present study are different from those in other studies. These measures may have been possible sources of bias in the present study.

Another limitation is that many studies have examined body image, eating patterns, BMI, and depression using samples composed mainly of Caucasian women, with African American women often underrepresented in research on this topic. More research on the relationship between weight and hypertension among urban African Americans is required before a conclusion can be drawn regarding BMI, hypertension, and symptoms of depression.

\section{Clinical Implications}

This study has implications for African American family members with a diagnosis of hypertension and the health practitioners responsible for their care. Screening hypertensive patients for depression is important when evaluating their care and prescribing treatment modalities to improve their well-being. Although diet and exercise are key elements that have been established as effective treatments in managing high blood pressure, psychosocial factors (eg, levels of stress and depression) may also contribute to increases in blood pressure. If health care providers examined stress and depression to the same extent as diet and exercise, additional patients with a dual diagnosis of hypertension and depression could receive appropriate treatment. After stress or depression levels are determined to be above average for a patient with hypertension, health care providers could refer these individuals to the appropriate resources (eg, social worker, psychologist, psychiatrist) for further evaluation and follow-up. Such referrals could decrease the need for expensive antihypertensive medications and routine treatment modalities that may be ineffective in those experiencing symptoms of depression. If practitioners treat the underlying problems of stress and depression first, then hypertension may have a better probability of being controlled.

\section{Future Research}

This study should be replicated with a larger heterogeneous sample to determine whether the findings are consistent across different ethnic groups. Research on this topic should include a holistic conceptual framework, such as the Neuman System Model, because it discusses how stress interacts with spiritual, sociocultural, developmental, physiologic, and psychological aspects of a person or family to alter health equilibrium. ${ }^{53}$ The Neuman System Model has been previously used in research linking parenting stressors, lifestyle behaviors, and hypertension management among African American caregivers. $^{2,13}$ Details of the theoretical model used in the parent study have been outlined in Nursing Research. ${ }^{44}$

Additional research should be conducted using a measure for physical activity that is less reliant on self-report to increase the objectivity in the variable. The present study's use of self-report for sodium intake could be replaced with a clinical measure that could provide a more accurate measure of sodium intake among participants. Depression, in future research, should use a measure that captures the multidimensional aspects of depression in a person's life. The use of a unidimensional measure of depression may not provide information on the specific type of depression that could be affecting hypertension among African American parents and grandparents.

Disclosure: Funding for this research was provided in part by Minority Supplement 3 ROI NRO 7682-02 S1, R01 NR07682-04, KL2 RR024987-01, P30 NR009000, 5-P30-AG015281-07.

\footnotetext{
REFERENCES

1 Dragan A, Akhtar-Danesh N. Relation between body mass index and depression: a structural equation modeling approach. BMC Med Res Methodol. 2007;7:17.

2 Taylor JY, Washington OGM, Artinian NT, et al. Predictors of parental stress among African American parents and grandparents. Issues Ment Health Nurs. 2007;28(4):373-387.

3 Collins R, Peto R, MacMahon S, et al. Blood pressure, stroke, and coronary heart disease. Part 2, Short-term reductions in blood pressure: overview of randomized drug trials in their epidemiological context. Lancet. 1990;335:827-838.

4 Hebert PR, Moser M, Mayer J, et al. Recent evidence on drug therapy of mild to moderate hypertension and decreased risk of coronary heart disease. Arch Intern Med. 1993;153:578-581.

5 Glover MJ, Greenlund KJ, Ayala C, et al.
}

Racial/ethnic disparities in prevalence, treatment, and control of hypertension-United States, 1999-2002. MMWR. 2005;54:7-9.

6 Burt VL, Cutler JA, Higgins M, et al. Trends in the prevalence, awareness, treatment, and control of hypertension in the adult US population. Data from the health examination surveys, 1960 to 1991. Hypertension. 1995;26:60-69.

7 Ayala C, Neff LJ, Croft JB, et al. Prevalence of self-reported high blood pressure awareness, advice received from health professionals, and actions taken to reduce high blood pressure among US adults-Healthstyles 2002. J Clin Hypertens (Greenwich). 2005;7(9):513-519.

8 Schnittker J. Chronic illness and depressive symptoms in late life. Soc Sci Med. 2005;60:13-23.

9 Artinian NT, Washington OW, Flack JM, et al. Depression, stress, and blood pressure in urban
African American women. Prog Cardiovasc Nurs. 2006;21:68-75.

10 Wu CY, Prosser R, Taylor JY. Association of symptoms of depression and social support on hypertension among urban African American women. Prog Cardiovasc Nurs. 2008. In press.

11 Butler FR, Zakari N. Grandparents parenting grandchildren: assessing health status, parental stress, and social supports. J Gerontol Nurs. 2005;31(3):43-54.

12 Taylor JY, Washington OGM, Artinian NT, et al. Urban hypertensive African American grandparents: stress, health and implications of child care. J Aging Ment Health. 2007;30(4):39-54.

13 Long JY. The relationship between parental attitudes and lifestyle behaviors among hypertensive African Americans caring for children and/or grandchildren. Doctoral Dissertation. Ann Arbor, MI 
UMI Publishing Company;2005. Copyright TX 6-046-201, United States Copyright Office.

14 Dilworth-Anderson P, Goodwin PY, Wallace Williams S. Can culture help explain the physical health effects of caregiving over time among African American caregivers? J Gerontol. 2004;59:S138-S145.

15 Wallace Williams S, Dilworth-Anderson P, Goodwin PY. Caregiver role strain: the contribution of multiple roles and available resources in African-American women. Aging Ment Health. 2003;7(2):103-112.

16 Rosamond W, Flegal K, Friday G, et al. Heart Disease and Stroke Statistics-2007 Update. A report from the American Heart Association Statistics Committee and Stroke Committee. Circulation. 2007;115(5):e69-e171.

17 Rutledge T, Hogan BE. A quantitative review of prospective evidence linking psychological factors with hypertension. Psychosom Med. 2002;64:758-766.

18 Yan LL, Liu K, Matthews KA, et al. Psychosocial factors and risk of hypertension: The coronary artery risk development in young adults (CARDIA) study. JAMA. 2003;290(16):2138-2148.

19 Reiff M, Schwartz S, Northridge M. Relationship of depressive symptoms to hypertension in a household survey in Harlem. Psychosom Med. 2001;63:711-721.

20 Jonas BS, Lando JF. Negative affect as a prospective risk factor for hypertension. Psychosom Med. 2000;62:188-196.

21 Minkler M, Fuller-Thomson E. Physical and mental health status of American grandparents providing extensive child care to their grandchildren. J Am Med Womens Assoc. 2001;56(4):199-205.

22 Musil CM, Ahmad M. Health of grandmothers: a comparison by caregiver status. J Aging Health. 2002;14(1):96-121.

23 Fuller-Thomson E, Minkler M. African American grandparents raising grandchildren: a national profile of demographic and health characteristics. Health Soc Work. 2000;25(2):109-118.

24 Whitley DM, Kelley SJ, Sipe TA. Grandmothers raising grandchildren: are they at increased risk of health problems? Health Soc Work. 2001;26(2):105-114

25 Jones Warren B. Examining depression among African-American women from a psychiatric mental health nursing perspective. Healthy Place.com Depression Community. http://www. healthyplace.com/communities/depression/ minorities_8.asp. Accessed October 1, 2007.
26 Johnston E, Johnson S, McLeod P, et al. The relation of body mass index to depressive symptoms. Can J Public Health. 2004;95(3):179-183.

27 White MA, Grilo CM. Ethnic differences in the prediction of eating and body image disturbances among female adolescent psychiatric inpatients. Int J Eat Disord. 2005;38:78-84.

28 World Health Organization. Report on a WHO Consultation, Technical Report Series, No. 894-Obesity: Preventing and Managing the Global Epidemic. Geneva, Switzerland: World Health Organization; 2000.

29 NICE issues guidance on surgery for morbid obesity. National Institute for Health and Clinical Excellence Web site. http://www.nice.org.uk/ page.aspx?o=34790. Accessed January 10, 2008.

30 Dong C, Sanchez LE, Rice RA. Relationship of obesity to depression: a family-based study. Int J Obes Relat Metab Disord. 2004;28:790-795.

31 Hatfield BD, Landers DM. Psychophysiology in exercise and sport research: an overview. Exerc Sport Sci Rev. 1987;15:351-387.

32 Rehor PR, Dunnagan T, Stewart C, et al. Alteration of mood state after a single bout of noncompetitive and competitive exercise programs. Percept Mot Skills. 2001;93(1):249-256.

33 Stewart KJ, Turner KL, Bacher AC, et al. Are fitness, activity, and fatness associated with health-related quality of life and mood disorders in older persons. J Cardiopulm Rehabil. 2003;23(2):115-121.

34 Flack J. Hypertension and African-Americans: customizing treatment options. Therapeutic Spotlight: A Self-Study Supplement to Clinician Reviews. 2003.

35 Lynch EW, Hanson MJ, eds. Developing Crosscultural Competence: A Guide for Working With Children and Their Families. 2nd ed. Baltimore, MD: Paul H. Brookes Publishing; 1998.

36 Son BK, Markovitz JH, Winders S, et al. Smoking, nicotine dependence, and depressive symptoms in the CARDIA Study. Effects of educational status. Am J Epidemiol. 1997;145(2):110-116.

37 Repetto PB, Caldwell CH, Zimmerman MA. A longitudinal study of the relationship between depressive symptoms and cigarette use among African American adolescents. Health Psychol. 2005;24(2):209-219.

38 Williams CD, Adams-Campbell LL. Addictive behaviors and depression among African Americans residing in a public housing community. Addict Behav. 2000;25(1):45-56.

39 Braszko JJ, Karwowska-Polecka W, Halicka D, et al. Captopril and enalapril improve cognition and depressed mood in hypertensive patients. $J$ Basic Clin Physiol Pharmacol. 2003;14(4):323-343.

40 Brown DR, Keith VM, eds. In and Out of Our Right Minds: The Mental Health of African American Women. New York, NY: Columbia University Press; 2003:23-25.

41 Taylor RJ, Chatters LM, Jackson JS. Religious and spiritual involvement among older African Americans, Caribbean Blacks, and Non-Hispanic Whites: findings for the national survey of American life. J Gerontol B Psychol Sci Soc Sci. 2007;62(4):S238-S250.

42 Giaquinto S, Spiridigliozzi C. Possible influence of spiritual and religious beliefs on hypertension. Clin Exp Hypertens. 2007;29(7):457-464.

43 Hixson KA, Gruchow HW, Morgan DW. The relationship between religiosity, selected health behaviors, and blood pressure among adult females. Prev Med. 1998;27(4):545-552.

44 Artinian NT, Flack JM, Nordstrom CK, et al. Effects of nurse-managed telemonitoring on blood pressure at 12-month follow-up among urban African Americans. Nurs Res. 2007;56(5):312-322.

45 Cohen J. Quantitative methods in psychology: a power primer. Psychol Bull. 1992;112:155-159.

46 Cohen S, Kamarck T, Mermelstein R. A globa measure of perceived stress. J Health Soc Behav. 1983;24:385-396.

47 Mendes de Leon CF, Dilillo V, Czajkowski S, et al; Enhancing Recovery in Coronary Heart Disease (ENRICHD) Pilot Study. Psychosocia characteristics after acute myocardial infarction: the ENRICHD pilot study. Enhancing Recovery in Coronary Heart Disease. J Cardiopulm Rehabil. 2001;21(6):353-362.

48 Mitchell PH, Powell L, Blumethal J, et al. A short social support measure for patients recovering from myocardial infarction. J Cardiopulm Rehabil. 2003;23:398-403.

49 Radloff LS. The CES-D scale: A self-report depression scale for research in the general population. Appl Psychol Meas. 1977;1:385-401.

50 The Seventh Report of the Joint Committee on Prevention, Detection, Evaluation, and Treatment of High Blood Pressure. JAMA. 2003;289:2560-2572.

51 Dabl Educational Trust. The British Hypertension Society and the Association for the Advancement of Medical Instrumentation. http://www.dableducational.com. Accessed October 31, 2005.

52 Block G. A review of validations of dietary assessment methods. Am J Epidemiol. 1982;115:492-505.

53 Neuman B, Fawcett J. The Neuman Systems Model. 4th ed. Stamford, CT: Appleton \& Lange; 2002. 